\title{
A forced marriage of IL-2 and PD-1 antibody nurtures tumor-infiltrating $T$ cells
}

\author{
Erin A. Holcomb ${ }^{1}$ and Weiping Zou ${ }^{1,2,3,4}$ \\ ${ }^{1}$ Graduate Program in Immunology, ${ }^{2}$ Department of Surgery, and ${ }^{3}$ Department of Pathology, University of Michigan School of Medicine, Ann Arbor, Michigan, USA. ${ }^{4}$ Center of Excellence for Cancer \\ Immunology and Immunotherapy, University of Michigan Rogel Cancer Center, Ann Arbor, Michigan, USA.
}

\begin{abstract}
IL-2 is a pleiotropic cytokine. In this issue of the $J C I$, Ren et al. report on the development of a low-affinity IL-2 paired with anti-PD-1 (PD-1-lalL-2) that reactivates intratumoral $\mathrm{CD}^{+} \mathrm{T}$ cells, but not $\mathrm{CD4}{ }^{+}$Treg cells. PD-1-lalL-2 treatment synergized with anti-PD-L1 therapy to overcome tumor resistance to immune checkpoint blockade (ICB) in tumor-bearing mice. Rejection of rechallenged tumors following PD-1-lalL-2 therapy demonstrated the establishment of a potent T cell memory response. Furthermore, PD-1-lalL-2 therapy manifested no obvious toxicity. These findings suggest the potential of PD-1-lalL-2 therapy in treating patients with cancer.
\end{abstract}

\section{Targeting IL-2 signaling} pathway for cancer therapy

IL-2 is produced primarily by activated $\mathrm{CD}^{+} \mathrm{T}$ cells and acts in a paracrine or autocrine fashion $(1,2)$. IL-2 receptor (IL-2R) signaling occurs through three subunits: alpha (CD25), beta (CD122), and gamma (CD132) (3). Intermediateaffinity dimeric IL-2 receptor consists of IL-2R $\beta$ and IL-2R $\gamma$ on naive $\mathrm{CD}^{+}$and $\mathrm{CD}^{+} \mathrm{T}$ cells, memory $\mathrm{T}$ cells, and natural killer (NK) cells. TCR engagement or IL-2 stimulation induces the expression of IL-2R $\alpha$ to form high-affinity trimeric IL-2 receptors that are highly expressed on Treg cells and recently activated effector T cells (4). IL-2 signaling has been an attractive immunotherapeutic target since IL-2 mediates effector $\mathrm{T}$ cell activation, including effector $\mathrm{CD}^{+} \mathrm{T}$ cells, which are vital for antitumor immunity. High-dose IL-2 was approved by the FDA in 1992 for treatment of certain types of cancer (5). However, IL-2 possesses a very short half-life and requires high dos- es to be effective, leading to toxicity and severe side effects, such as inflammation and vascular leak syndrome (6). Alternatively, low doses of IL-2 preferentially target IL-2R $\alpha$ on Treg cells, restricting the immune response, and are associated with poor prognosis in patients with cancer $(7,8)$. Therefore, methods to target certain $\mathrm{T}$ cell subsets while reducing Treg cell binding have been a recent focus in the field of IL-2 therapy.

\section{Manipulation of T cell phenotype by IL-2 therapy}

To effectively manipulate effector $\mathrm{T}$ cells and reduce side effects of high-dose IL-2, IL-2 variants have been developed to stimulate specific $\mathrm{T}$ cell subsets through selective targeting of certain IL-2R chains. One strategy has been to introduce mutations in IL-2 to create mutants with preferential IL-2R chain binding. Mutants with reduced IL-2R $\beta$ binding have been shown to target high-affinity IL-2 receptor expressed on effector T
Related Article: https://doi.org/10.1172/JCI153604

Conflict of interest: WZ serves as a scientific consultant or board member at NGM Biopharmaceuticals Inc., Roivant, NextCure, CStone, CrownBio, and Intergalactic Therapeutics.

Copyright: (c) 2022, Holcomb et al. This is an open access article published under the terms of the Creative Commons Attribution 4.0 International License.

Reference information: / Clin Invest. 2022;132(3):e156628. https://doi.org/10.1172/JCI156628. cells (Figure 1). These mutants have also exhibited reduced toxicity, possibly due to decreased binding of intermediate-affinity receptors on NK cells that lack IL-2R $\alpha$ $(1,9)$. STK-012, a partial IL-2 agonist produced by Synthekine, employs a similar strategy by selectively binding IL-2R $\alpha$ and IL-2R $\beta$ subunits, but not IL-2R $\gamma$. Effector $\mathrm{T}$ cells that may be specific for tumor epitopes can thus expand and readily attack the tumor while avoiding NK cell stimulation (10). However, undesirable Treg cell expansion remains a concern due to high IL-2R $\alpha$ expression on Treg cells (7, 8). To address this issue, IL-2 mutants with reduced binding to IL-2R $\alpha$ have also been generated. The cytokine company Nektar has engineered an IL-2 mutant with a bias toward IL-2R $\beta$ and IL-2R $\gamma$, rather than IL-2R $\alpha$, to reduce Treg cell binding (10). H9, an IL-2 superkine (sumIL-2) with enhanced IL-2R $\beta$ binding without the need for IL-2R $\alpha$, was shown to increase expansion of cytotoxic memory $\mathrm{T}$ cells and NK cells while decreasing that of Treg cells (11). Interestingly, H9T, an engineered H9-based partial agonist with further reduced binding to IL-2R $\gamma$, was also recently shown to promote $\mathrm{CD} 8^{+} \mathrm{T}$ cell proliferation that maintained a stemlike memory state and mediated greater antitumor immunity (12).

To enhance the activity of IL-2 in vivo and limit toxicity by reducing the necessary dose, IL-2 therapy has been combined with anti-IL-2 monoclonal antibodies $(\mathrm{mAb})$. Interestingly, various anti-IL-2 mAbs differentially stimulate different immune cell subsets. Anti-mouse IL-2 mAbs S4B6 and JES6-5, as well as antihuman IL-2 mAb MAB602, complexed with recombinant IL-2, selectively stimulate memory $\mathrm{CD} 8^{+}$cells and NK cells in vivo to improve IL-2 cancer therapy (Figure 1) (13). On the other hand, anti-IL-2 mAb JES6-1 inhibits proliferation of $\mathrm{CD} 8^{+}$ cells and NK cells yet maintains its ability to activate Treg cells and has been impli- 


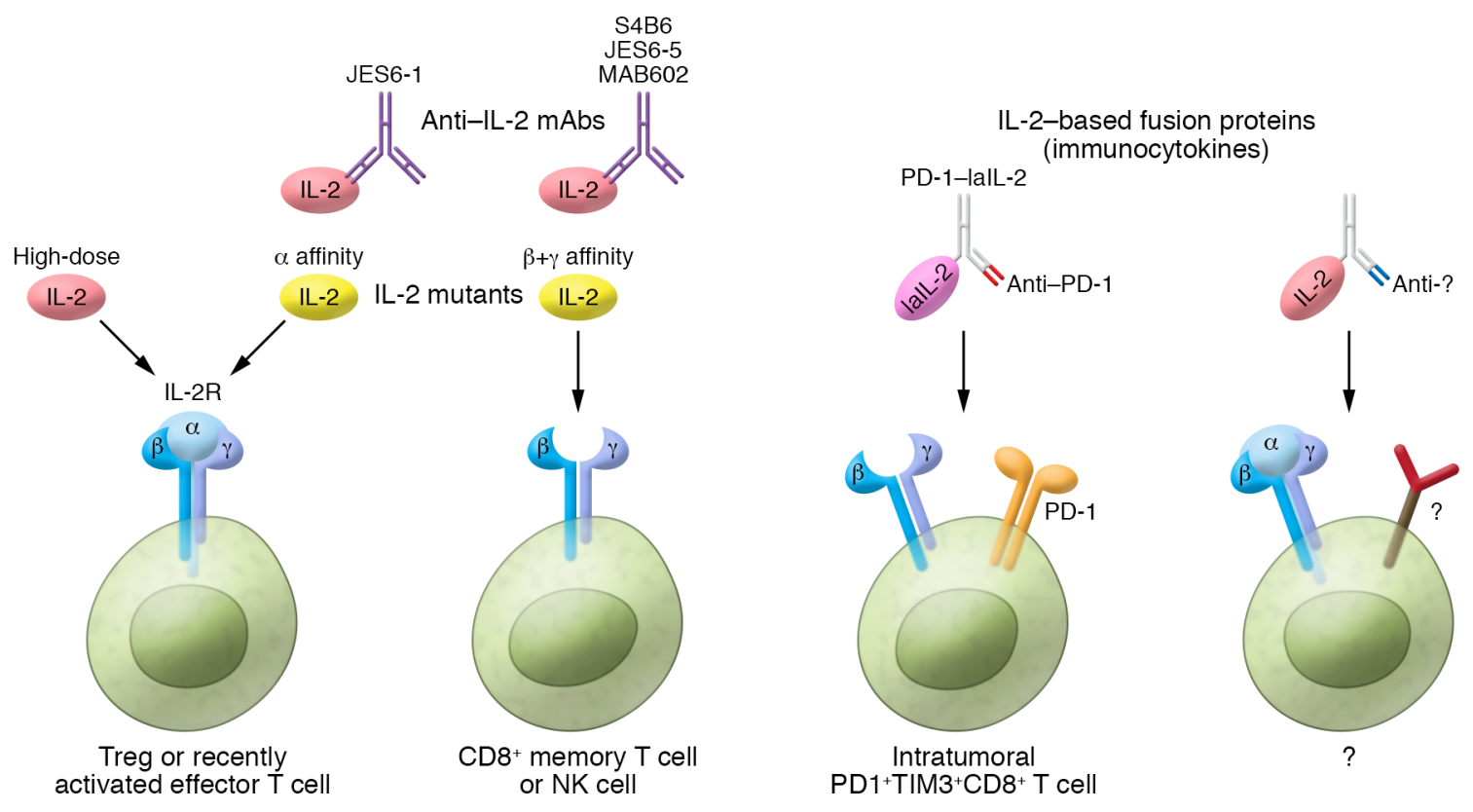

Figure 1. Targeting IL-2 signaling for cancer therapy. High-dose IL-2 may preferentially target high-affinity IL-2R present on Treg cells and recently activated effector T cells. Recent strategies to target IL-2 signaling for cancer therapy include mutant IL-2 with affinity toward different IL-2R chains (alpha, or beta and gamma). Mutant IL-2 with affinity toward IL-2R $\alpha$ is used to target Treg cells or recently activated effector T cells. Meanwhile, mutant IL-2 with affinity toward IL-2R $\beta$ or IL-2R $\gamma$ subunits, rather than IL-2R $\alpha$, has been shown to target CD8 $8^{+}$memory T cells and NK cells with reduced binding to Treg cells. Combination of IL-2 therapy with various anti-IL-2 mAbs also differentially stimulates specific immune cell subsets. IL-2-based fusion proteins bound to antigen-specific antibodies (immunocytokines) allow for targeted delivery of IL-2 to cells/tissues expressing a protein of interest. PD-1-lalL-2, developed by Ren et al. (20), consists of low-affinity IL-2 (lalL-2) linked to an anti-PD-1 antibody. PD-1-lalL-2 selectively reactivates intratumoral PD-1 ${ }^{+}$TIM-3 $3^{+}$CD $8^{+}$T cells to enhance antitumor activity. In the future, additional IL-2-based fusion proteins may be engineered to target certain cells of interest in various disease contexts.

cated as a potential treatment for autoimmune disease (14). Binding of these various mAbs to certain regions of IL-2, therefore blocking IL-2 binding to specific IL-2R chains, may explain these contrasting cell type affinities $(1,2)$.

IL-2-based fusion proteins are another IL-2 therapy strategy with a multitude of current preclinical and clinical trials (15, 16). Fusion of IL-2 to a fragment crystallizable $(\mathrm{Fc})$ region has proven to be beneficial due to increased half-life, complement activation, and induction of antibodydependent cellular cytotoxicity (ADCC) toward Treg cells (17-19). Furthermore, fusion of IL-2 to antigen-specific antibodies (termed an immunocytokine) allows for targeted delivery of IL-2 to cells and tissues expressing a protein of interest. Numerous IL-2 immunocytokines have been developed to target tumor-associated antigens expressed by cancer cells and their surrounding tissue (16). IL-2 is therefore honed to tumor tissues to enact its function. However, this strategy still lacks the ability to specifically target effector $\mathrm{T}$ cells within the tumor that are pertinent to anticancer immunity.
Targeting intratumoral effector T cells with IL-2 and anti-PD-1 therapy

Ren et al. (20) addressed this intratumoral $\mathrm{T}$ cell targeting gap by engineering an immunocytokine fusion protein consisting of low-affinity IL-2 (laIL-2) linked to an anti-PD-1 antibody (PD-1-laIL-2). laIL-2 exhibits reduced binding to IL-2R $\alpha$ and IL-2R $\beta$ to diminish unfavorable Treg cell binding in the tumor and periphery. Meanwhile, PD-1 is highly expressed on tumor-infiltrating $\mathrm{CD}^{+} \mathrm{T}$ cells. As a result, PD-1-laIL-2 possessed elevated avidity toward intratumoral $\mathrm{CD} 8^{+} \mathrm{T}$ cells, rather than Treg cells or peripheral $\mathrm{CD}^{+}$and $\mathrm{CD} 8^{+} \mathrm{T}$ cells. This specificity not only reduced the systemic toxicity, but also enhanced tumor control in A2O and MC38 tumor models, as well as A375 tumor-bearing humanized mice. In addition, PD-1-laIL-2 in combination with anti-PD-L1 therapy overcame tumor resistance to PD-L1 blockade therapy. Notably, this effect was dependent on intratumoral $\mathrm{CD} 8^{+} \mathrm{T}$ cells, whose proliferation was selectively induced by PD-1laIL-2. Further investigation revealed that
PD-1-laIL-2 seemed to selectively target intratumoral $\mathrm{PD}-1^{+} \mathrm{TIM}-3^{+} \mathrm{CD} 8^{+} \mathrm{T}$ cells, which are usually described as a functionally exhausted and/or terminally differentiated $\mathrm{T}$ cell subset. Therefore, PD-1laIL-2 could reactivate PD- $1^{+} \mathrm{TIM}-3^{+} \mathrm{CD} 8^{+}$ $\mathrm{T}$ cells to enhance antitumor activity (Figure 1). Tumor rechallenge resulted in spontaneous rejection in tumor-bearing mice previously treated with PD-1-laIL-2. This effect was also dependent on the presence of $\mathrm{CD} 8^{+} \mathrm{T}$ cells, indicating these rejuvenated $\mathrm{T}$ cells are tumor antigenspecific and can mediate a strong memory response. These promising results suggest that PD-1-laIL-2 therapy may bring clinical benefits to patients with cancer.

Address correspondence to: Weiping Zou, Departments of Surgery and Pathology, University of Michigan Medical School, 109 Zina Pitcher Place, Ann Arbor, Michigan 48109, USA. Phone: 734.763.6402; Email:wzou@med.umich.edu.

\footnotetext{
1. Boyman O, et al. Potential use of IL-2/anti-IL-2 antibody immune complexes for the treatment of cancer and autoimmune disease. Expert Opin Biol Ther. 2006;6(12):1323-1331.
} 
2. Létourneau S, et al. IL-2/anti-IL-2 antibody complexes show strong biological activity by avoiding interaction with IL-2 receptor alpha subunit CD25. Proc Natl Acad Sci U S A. 2010;107(5):2171-2176.

3. Wang X, et al. Structure of the quaternary complex of interleukin-2 with its alpha, beta, and gammac receptors. Science. 2005;310(5751):1159-1163.

4. Létourneau S, et al. IL-2- and CD25-dependent immunoregulatory mechanisms in the homeostasis of T-cell subsets. J Allergy Clin Immunol. 2009;123(4):758-762.

5. Rosenberg SA. IL-2: the first effective immunotherapy for human cancer. JImmunol. 2014;192(12):5451-5458.

6. Pachella LA, et al. The toxicity and benefit of various dosing strategies for interleukin-2 in metastatic melanoma and renal cell carcinoma. JAdv Pract Oncol. 2015;6(3):212-221.

7. Wei S, et al. Interleukin-2 administration alters the CD4+FOXP3+ T-cell pool and tumor trafficking in patients with ovarian carcinoma. Cancer Res. 2007;67(15):7487-7494.
8. Kryczek I, et al. Cutting edge: Th17 and regulatory $\mathrm{T}$ cell dynamics and the regulation by $\mathrm{IL}-2$ in the tumor microenvironment. JImmunol. 2007;178(11):6730-6733.

9. Shanafelt AB, et al. A T-cell-selective interleukin 2 mutein exhibits potent antitumor activity and is well tolerated in vivo. Nat Biotechnol. 2000;18(11):1197-1202.

10. Garber K. Synthekine: beyond cytokines [published online June 9, 2021]. Nat Biotechnol. https://doi.org/10.1038/d41587.021.00006-6.

11. Levin AM, et al. Exploiting a natural conformational switch to engineer an interleukin-2 'superkine'. Nature. 2012;484(7395):529-533.

12. Mo F, et al. An engineered IL-2 partial agonist promotes $\mathrm{CD}^{+} \mathrm{T}$ cell stemness. Nature. 2021;597(7877):544-548.

13. Boyman O, et al. Selective stimulation of T cell subsets with antibody-cytokine immune complexes. Science. 2006;311(5769):1924-1927.

14. Webster KE, et al. In vivo expansion of T reg cells with IL-2-mAb complexes: induction of resistance to EAE and long-term acceptance of islet allografts without immunosuppression. J Exp
Med. 2009;206(4):751-760.

15. Mullard A. Restoring IL-2 to its cancer immunotherapy glory. Nat Rev Drug Discov. 2021;20(3):163-165.

16. MacDonald A, et al. Interleukin 2-based fusion proteins for the treatment of cancer. JImmunol Res. 2021;2021:7855808.

17. Hsu EJ, et al. A cytokine receptor-masked IL2 prodrug selectively activates tumor-infiltrating lymphocytes for potent antitumor therapy. Nat Commun. 2021;12(1):2768.

18. Zhu EF, et al. Synergistic innate and adaptive immune response to combination immunotherapy with anti-tumor antigen antibodies and extended serum half-life IL-2. Cancer Cell. 2015;27(4):489-501.

19. Vazquez-Lombardi R, et al. Potent antitumour activity of interleukin-2-Fc fusion proteins requires $\mathrm{Fc}$-mediated depletion of regulatory T-cells. Nat Commun. 2017;8:15373.

20. Ren Z, et al. Selective delivery of low-affinity IL-2 to PD-1+ T cells rejuvenates antitumor immunity with reduced toxicity.J Jlin Invest. 2021;132(3):e153604. 